\title{
Interferometric vs wavelength selective optical fiber sensors for cryogenic temperature measurements
}

\author{
V. DeMiguel-Soto $*^{\mathrm{a}}$, D. Leandro ${ }^{\mathrm{a}}$, A. Lopez-Aldaba ${ }^{\mathrm{a}}$, J.J. Beato-López ${ }^{\mathrm{b}}$, J.I. Pérez-Landazábal ${ }^{\mathrm{b}, \mathrm{c}}$, \\ J.-L. Auguste ${ }^{\mathrm{d}}$, R. Jamier ${ }^{\mathrm{d}}$, P. Roy ${ }^{\mathrm{d}}$, M. Lopez-Amo ${ }^{\mathrm{a}}$ \\ ${ }^{a}$ Universidad Pública de Navarra, Dept. of Electrical and Electronic Engineering, Campus de \\ Arrosadia S/N, E-31006, Pamplona, Spain; ' Universidad Pública de Navarra, Dept. of Physics, \\ 31006 Pamplona, Spain; ${ }^{c}$ Universidad Pública de Navarra, Institute for Advanced Materials \\ (INAMAT), 31006 Pamplona, Spain; ${ }^{d}$ Xlim, Fibre Photonics Department, UMR CNRS/University \\ of Limoges 7252, 123 Avenue Albert Thomas, 87060 Limoges Cedex, France. \\ *veronica.demiguel@unavarra.es; phone +34 94816 9841; fax +34948 16 9720;
}

\begin{abstract}
In this work, a preliminary study of the behavior of two different interferometric fiber optic sensors and two different wavelength selective fiber optic sensors is performed. A photonic cristal fiber Fabry-Pérot interferometer, a Sagnac interferometer, a commercial fiber Bragg grating (FBG) and a $\pi$-phase shifted fiber Bragg grating interrogated in a random distributed feedback fiber laser are analyzed. A comparison of their sensitivities and resolutions is carried out to analyze their performance as sensors for cryogenic temperatures, taking into account their advantages and drawbacks.
\end{abstract}

Keywords: cryogenic temperature, interferometric sensor, optical fiber sensor, random distributed feedback fiber lasers

\section{INTRODUCTION}

Systems operating at cryogenic temperatures are becoming more and more important in the energy sector, transportation, and medicine technology. At these temperatures, providing continuous monitoring of the temperature to ensure a safe operation of these systems becomes crucial. However, temperature monitoring at cryogenic temperatures is significantly more challenging than equivalent measurements at room temperature. For most cryogenic applications, resistive, diode, and thermocouple sensors are the most extended [1]. Nevertheless, fiber optic sensors offer important advantages in cryogenic environments over conventional approaches because of their compactness, lightweight, immunity to electromagnetic interference, ability to operate in harsh environments and their multiplexing capability, which decreases the total cost of the system [2]. The main drawback of fiber optic sensors is their low intrinsic thermal sensitivity at low temperatures [3]. In fact, the low thermal expansion coefficient of silica limits the temperature sensitivity of the sensors at cryogenic temperatures. However, many works have reported the use of fiber Bragg gratings (FBG) for cryogenic measurements, embedding in or bonding the FBG to substrates in order to enhance their temperature sensitivity. These substrates, such as poly methyl methacrylate (PMMA) or Teflon, have larger thermal expansion coefficients than silica fibers [4]. Metal-coated fiber Bragg Gratings [3] and long period gratings inscribed in different types of fiber [5] have been also tested. While the enhancement of FBG temperature sensitivity is significant using these techniques, the flexibility of the optical fiber is sacrificed because of the need for bonding to a rigid substrate.

As far as we are concerned, it has not been previously reported in the literature the use of other types of fiber optic sensors at cryogenic temperatures. In this work, a preliminary study of the behavior of two different interferometers and two different wavelength-selective fiber optic sensors is carried out, comparing their capabilities and weaknesses. The feasibility of using the different approaches to measure cryogenic temperatures is analyzed by comparing their main advantages and drawbacks.

\section{EXPERIMENTAL SETUP AND PRINCIPLE OF OPERATION}

\subsection{Experimental setup}

The setup used to perform the cryogenic measures consists of an expandable Polystyrene (EPS) box and a solid copper cylinder, which has good thermal conductivity, located inside the box (Fig. 1). Four different fiber optic sensors were analyzed in the work. Two fiber Bragg gratings were employed: a commercial fiber Bragg grating (FBG) and a $\pi$-phase

25th International Conference on Optical Fiber Sensors, edited by Youngjoo Chung, Wei Jin,

Byoungho Lee, John Canning, Kentaro Nakamura, Libo Yuan, Proc. of SPIE Vol. 10323,

1032337 - (c) 2017 SPIE · CCC code: 0277-786X/17/\$18 · doi: 10.1117/12.2264889 
shifted fiber Bragg grating (PSFBG) interrogated in a random distributed feedback fiber laser (RDFB-FL) configuration. Additionally, two interferometric approaches were used: a photonic crystal fiber Fabry-Pérot interferometer and Sagnac interferometer. The first three sensors were fixed on top of the copper cylinder, directly in contact with it in order to have an effective transmission of the temperature. The Sagnac interferometer was wrapped around the top part of the cylinder because of its length. Together with the sensors, a thermocouple type $\mathrm{K}$ was placed inside to provide a temperature reference in real-time. The thermocouple was connected to a data acquisition system where the temperature data was stored every second. Both interferometric sensors and the FBG were connected to a commercial fiber Bragg grating interrogator, Smartec SM125, with and spectral range from $1510 \mathrm{~nm}$ to $1590 \mathrm{~nm}$, a resolution of $5 \mathrm{pm}$ and a scan frequency of $1 \mathrm{~Hz}$.
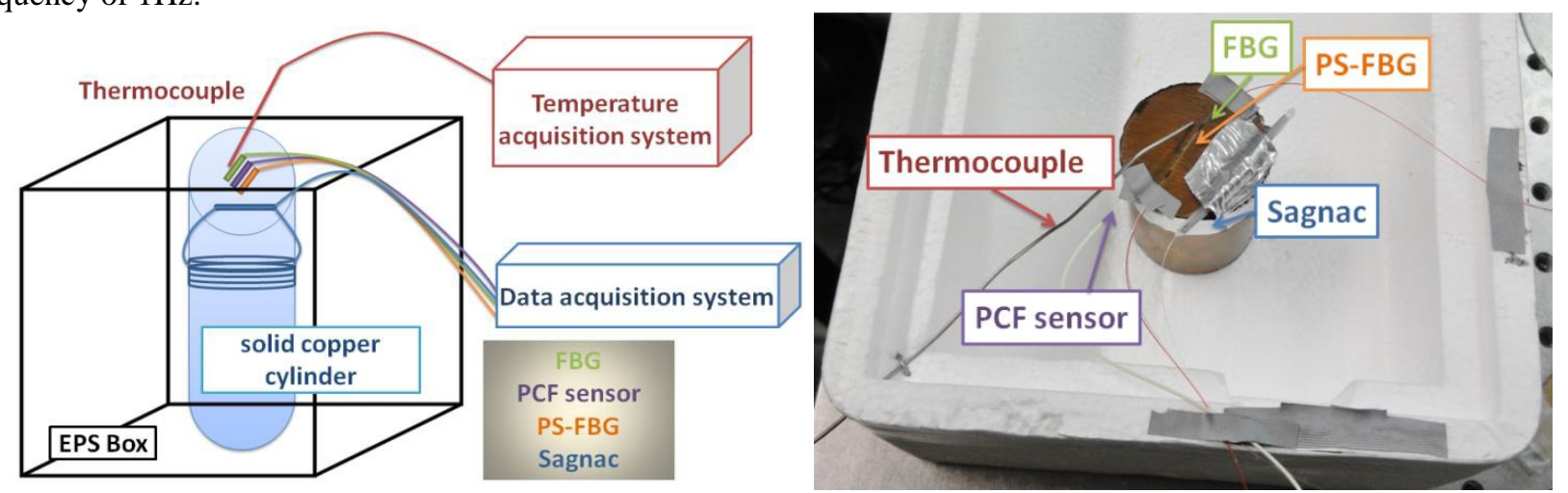

Figure 1.a) Schematic setup and b) real photograph of the setup

\subsection{Interferometric fiber optic sensors}

Firstly, the PCF Fabry-Pérot interferometer used in the experiment consists of a FP cavity, which was made by splicing a standard single mode fiber to one side of a four-bridge double-Y-shape-core micro structured optical fiber (MOF). The MOF was cleaved at the other end, leaving the cavity with a length of $570 \mu \mathrm{m}[6]$.

The Sagnac interferometer used in the experiment consists of a high-birefringence fiber loop mirror fully made of polarization-maintaining (PM) Panda fiber which design is based on [7]. Two PM fibers act as communications channels $(0.3 \mathrm{~m}$ long each) while the sensing fiber, $0.45 \mathrm{~m}$ long, operates as the sensor itself. The communication PM fibers are fused to the sensing fiber with a $45^{\circ}$ rotation angle offset so their sensing contributions are suppressed, being only detected the birefringence changes on the sensing fiber. Additionally, as in [7], the use of an all-PM setup eliminates the need of polarization control inside the cavity and improves the stability and resolution of the system.

The interrogation technique used in both interferometric sensors is based on the fast Fourier transform (FFT) of the optical spectrum as in [8] due to the superior resolution achieved comparing to conventional techniques [7]. The FFT was computed using Matlab every second, giving real-time information of the sensor system. To monitor the temperature changes, the FFT phase evolution of the main component generated by each interferometer was tracked in the FFT amplitude spectrum.

\subsection{Wavelength selective fiber optic sensors}

Regarding the commercial FBG (centered at $1547.2 \mathrm{~nm}$ at room temperature), its wavelength shift was straightforwardly monitored each second with the FBG interrogator.

The PSFBG sensor forms part of a forward pumped RDFB-FL [9] acting both as a filter and as a sensor as it is showed in [10]. In order to measure the wavelength shift of the PSFBG, the heterodyne detection technique was utilized, due to benefit from the higher precision of electrical devices compared to optical spectrum analyzers. The beating signal of the output of the laser with an external tunable laser source was detected and then displayed at an electrical spectrum analyzer (ESA), tracking the displacement of the beating signal every degree Celsius.

\subsection{Cooling process}

In order to decrease the temperature of the sensors low enough, a simple technique using pure liquid nitrogen was employed. The EPS box was partially filled up with liquid nitrogen, so that the liquid did not reach the sensors and it gradually decreased the temperature of the copper cylinder and therefore the sensors. After adding the nitrogen, the box 
was covered. A stable temperature of $-160^{\circ} \mathrm{C}$ was reached using this method. Although liquid nitrogen's boiling temperature is $-195.8^{\circ} \mathrm{C}$ at one atmosphere, the copper cylinder did not reach such temperature. Temperature might be further lowered by improving the isolation of the setup. It is worth saying that this process is very simple but cannot reach pure cryogenic temperatures. However, it was used to obtain preliminary results of the feasibility of the different sensing approaches. It is expected to extend the work by using other techniques for reaching lower temperatures.

\section{EXPERIMENTAL RESULTS}

The behavior of four different types of sensors at cryogenic temperatures was experimentally studied. The results of the sensor's response versus temperature measured by the thermocouple are shown in Fig.2 for both interferometric and wavelength selective optical fiber sensors.
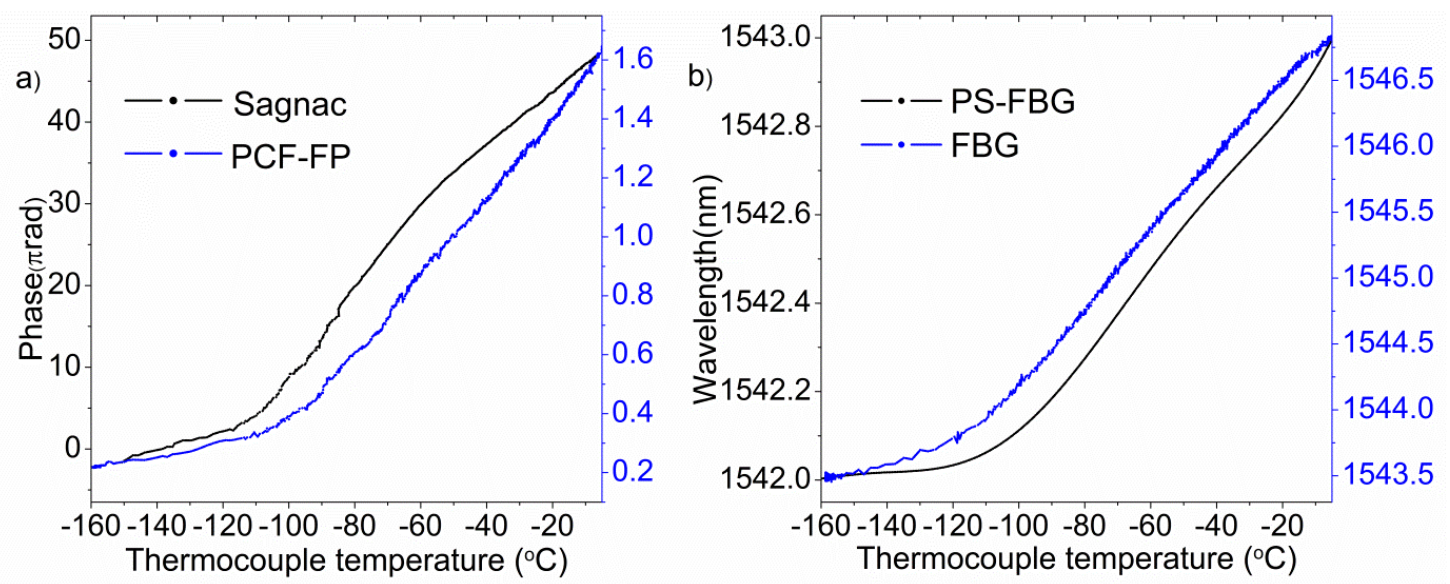

Figure2. a) Interferometric and b) wavelength selective optical fiber sensors cryogenic measurements

The measured data is represented as a function of the temperature measured with a thermocouple type $\mathrm{K}$, which has a standard limit error of $\pm 2.2^{\circ} \mathrm{C}$. A linear behavior is observed in every sensor in the temperature range above $-100^{\circ} \mathrm{C}$. In the case of the Sagnac interferometer, it seems that there are two different linear trends, probably due to the slow response time and the coupling of some axial strain to the fiber, generated by the expansion of the cylinder with temperature. However, below $-100{ }^{\circ} \mathrm{C}$, the slope of the curve decreases exponentially, reducing the sensitivity of the sensor. This fact was expected since the coefficient of thermal expansion of the fiber below $-100^{\circ} \mathrm{C}$ decrease faster [3], causing a change in the slope of the measurements. This leads to lower sensitivities and resolution of the measurements. The response time of the sensors was also studied, showing a response time that, as expected, is related to the size of the sensor and the presence/absence of coating. A summary of all the relevant measured data is reflected in Table 1.

\begin{tabular}{|c|c|c|c|c|}
\hline Type of sensor & Sensor & $\begin{array}{c}\text { Sensitivity above } \\
\mathbf{- 1 0 0}\end{array}$ & $\begin{array}{c}\text { Resolution above } \\
\mathbf{- 1 0 0}\end{array}$ & Response time \\
\hline \multirow{2}{*}{$\begin{array}{c}\text { Wavelength } \\
\text { selective }\end{array}$} & Commercial FBG & $28.39 \mathrm{pm} /{ }^{\circ} \mathrm{C}$ & $1.76^{\circ} \mathrm{C}$ & High \\
\cline { 2 - 5 } & PSFBG random laser & $9.2 \mathrm{pm} /{ }^{\circ} \mathrm{C}$ & $0.21^{\circ} \mathrm{C}$ & High \\
\hline \multirow{2}{*}{ Interferometric } & PCF Fabry-Pérot & $0.0134 \pi \mathrm{rad} /{ }^{\circ} \mathrm{C}$ & $1.49^{\circ} \mathrm{C}$ & Medium \\
\cline { 2 - 5 } & Sagnac & $0.4023 \pi \mathrm{rad} /{ }^{\circ} \mathrm{C}$ & $0.1^{\circ} \mathrm{C}$ & Very high \\
\hline
\end{tabular}

Table 1. Comparison of the sensors

On the one hand, when comparing the sensitivities and the response time of the interferometric sensors, the Sagnac turns out to have higher sensitivity, higher resolution and higher response time than the PCF-FP. This is due to the fact that the Sagnac has a fiber length very long compared to the PCF interferometer, which can almost be considered as a punctual sensor. Accordingly, resolution using the Sagnac interferometer is significantly higher. 
On the other hand, the PSFBG used in a RDFB-FL configuration presents less sensitivity than the FBG, which is an intrinsic property of the gratings used in the experiment but it is not related with the interrogation systems employed. However, even with a three times smaller sensitivity, the interrogation technique using the narrow random laser achieves more than eight times higher resolution. That is because of the outstanding stability properties of random lasers combined with the measurement technique in the electrical domain [9] [10]. The sensitivity of the FBG is expected to decrease significantly below $-160^{\circ} \mathrm{C}$, so the error in the measurement using a commercial interrogator would be very high. Although the PSFBG would also reduce its sensitivity as the FBG do, lower temperatures are expected to be measured properly using this method due to the high resolution. It should be noted that combining this technique with coatings and substrates applied to the gratings [3] [4] could considerably improve the resolution achieved at ultra-low temperatures. After several measuring tests, it is worth mentioning the fragility of the PCF-FP sensor, which got broken once during one measuring test, while the rest of the sensors remained unaffected after all the measurement process.

\section{CONCLUSIONS}

In this work, a preliminary study of the behaviour of four different fiber optic sensors is performed at cryogenic temperatures. In this regard, two interferometric setups (a PCF-FP and a Sagnac interferometer) and two different selective wavelength fiber optic sensors (a commercial FBG and a PSFBG in a RDFB-FL configuration) are examined. Their sensitivities and resolutions have been compared and it has been proved that they can be used to measure temperatures above $-160^{\circ} \mathrm{C}$. In further work, the performance of the sensors will be analyzed at lower temperatures in a more detailed study, being particularly promising the setups based on the random laser and the all-PM Sagnac interferometer due to the high resolution achieved.

\section{ACKNOWLEDGEMENTS}

Financial support from the Spanish Comisión Interministerial de Ciencia y Tecnología within projects TEC2013-47264C2-2-R and TEC2016-76021-C2-1-R and the FEDER funds are acknowledged.

\section{REFERENCES}

[1] Yeager, C. J., Courts, S. S. "A review of cryogenic thermometry and common temperature sensors," IEEE sensors journal, 1(4), 352-360 (2001).

[2] Grattan, K. T. V., Sun, T., "Fiber optic sensor technology: an overview," Sensors and Actuators A: Physical, 82(1), 40-61 (2000).

[3] Rajini-Kumar, R., Suesser, M., Narayankhedkar, K. G., Krieg, G., Atrey, M. D., "Performance evaluation of metal-coated fiber Bragg grating sensors for sensing cryogenic temperature," Cryogenics, 48(3), 142-147 (2008).

[4] Mizunami, T., Tatehata, H., Kawashima, H., "High-sensitivity cryogenic fibre-Bragg-grating temperature sensors using Teflon substrates," Measurement Science and Technology, 12(7), 914 (2001).

[5] Martins, R., Caldas, P., Teixeira, B., Azevedo, J., Monteiro, J., Belo, J. H., Araújo, J. P.,Santoss, J.L., Rego, G., "Cryogenic temperature response of reflection-based phase-shifted long-period fiber gratings," Journal of Lightwave Technology, 33(12), 2511-2517 (2015).

[6] Lopez-Aldaba, A., Pinto, A. M. R., Lopez-Amo, M., Frazão, O., Santos, J. L., Baptista, J. M., Baierl, H., Auguste, J.L., Jamier, R., Roy, P., "Experimental and Numerical Characterization of a Hybrid Fabry-Pérot Cavity for Temperature Sensing," Sensors, 15(4), 8042-8053 (2015).

[7] Leandro, D., Bravo, M., Lopez-Amo, M., "High resolution polarization independent high-birefringence fiber loop mirror sensor," Optics express, 23(24), 30985-30990 (2015).

[8] Leandro, D., Bravo, M., Ortigosa, A., Lopez-Amo, M., "Real-time FFT analysis for interferometric sensors multiplexing," Journal of Lightwave Technology, 33(2), 354-360. (2015).

[9] Turitsyn, S. K., Babin, S.A., El-Taher, A. E., Harper, P., Churkin, D. V., Kablukov, S.I., Ania-Castañon, J. D., Karalekas, V., Podivilov, E. V., "Random distributed feedback fibre laser," Nature Photonics, 4(4), 231-235 (2010).

[10] Leandro, D., deMiguel-Soto, V., Lopez-Amo. M., "High-resolution Sensor System Using a Random Distributed Feedback Fiber Laser," Journal of Lightwave Technology, 34(19), 4596-602 (2016). 